\title{
Comparison of acromio-axillo-suprasternal notch index with upper lip bite test and ratio of height to thyromental distance for prediction of difficult intubation: a prospective study
}

\author{
Juhi Bhaktavar ${ }^{1}$ Poonam Gupta ${ }^{2 *}$ \\ Senior Registrar ${ }^{1}$, Consultant and Professor ${ }^{2 *}$, Department of Anaesthesia and Intensive care, \\ Vardhman Mahavir Medical Collage and Safdarjang Hospital, New Delhi -110029, India.
}

\begin{abstract}
Preoperative evaluation of airway helps anaesthesiologists to make a strategy to secure airway to prevent life-threatening complications. The aim of the study was to compare the acromioaxillo-suprasternal notch index (AASI), the ratio of height to thyromental distance (RHTMD) and the upper-lip-bite test (ULBT) to predict difficult laryngeal visualization preoperatively.
\end{abstract}

Method: A total of 240 adult patients in American Society Anaesthesiologist physical status (ASA) I and II requiring general anaesthesia for routine surgery were enrolled. The airway was evaluated on the acromio-axillo-suprasternal notch index (AASI), upper lip bite test (ULBT), and the ratio of height to thyromental distance (RHTMD) for all enrolled patients. An experienced anaesthesiologist, not aware of the recorded preoperative airway evaluation, performed the laryngoscopy and laryngoscopic view was graded as per Cormack and Lehane classification. Primary objective was to assess the efficacy of AASI for prediction of the difficult airway. Secondary objective was to compare it with the RHTMD and ULBT.

Results: Difficult visualization of the larynx (DVL i.e: Cormack Lehane III and IV) was observed in 33 patients. Higher sensitivity 93.94\%, specificity 97.58\%, PPV 86.1\%, diagnostic accuracy $(97.08 \%)$ with low false positive (5) was for AASI as compared to the ULBT with the sensitivity of $42.4 \%$, specificity of $87.7 \%$, PPV $35 \%$ and diagnostic accuracy $81.25 \%$ and the corresponding value for the RHTMD was $75.76 \%, 47.34 \%, 18.66 \%$ and $51.25 \%$ respectively. Thus suggest, AASI to be superior to the RHTMD and the ULBT in the prediction of difficult visualization of the larynx. The area under the curve for the receiver operating curve (AUC of ROC) of AASI $(0.965,95 \% \mathrm{CI}=0.93-0.98 ; \mathrm{p}=<0001$ ) was significantly higher than the ULBT and RHTMD $(0.720,95 \% \mathrm{CI}=0.658-0.776 ; \mathrm{p}=<000.1)$ and $(0.576,95 \% \mathrm{CI}, 0.511-0.639 ; \mathrm{p}=<0.133)$ further, testify AASI to be superior to RHTMD and ULBT in the prediction of difficult intubation The best cut-off value for the AASI $>0.49 \mathrm{cms}$, RHTMD $<21 \mathrm{cms}$ and for ULBT $>2$ were endorsed for predicting difficulty in endotracheal intubation

Conclusion: Preoperative assessment valueof AASI $>0.49$ was found to be a good and reliable predictor for difficult visualization of the larynx.

Keywords: Difficult intubation; airway assessment tests; acromio-axillo-suprasternal notch index; upper-lip bite test; ratio of height to thyromental distance

\section{Introduction}

Unanticipated difficult or failed intubation put anaesthesiologists in a scary situation of lifethreatening complications such as hypoxic brain
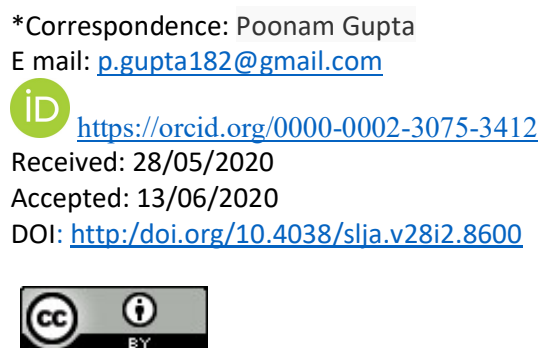

damage and death. ${ }^{1}$ Preoperative prediction of difficult laryngoscopy thus plays a paramount role in airway management as proper planning and strategies may decrease the morbidity or mortality. ${ }^{2,3}$

Acromio-axillo suprasternal notch index $(\mathrm{AASI})^{4}$ is an easy to perform bedside test for predicting difficulty in intubation. But little data is available comparing the AASI with other indices. Upper lip bite test (ULBT) and the ratio of height to thyromental distance (RHTMD) are claimed to have high predictability. ${ }^{5,6}$ Therefore the aim of this study was to evaluate the 
efficacy of AASI for assessing difficult laryngoscopy as an objective and comparing AASI with RHTMD and ULBT as a secondary objective.

\section{Method}

After approval from the hospital ethics committee, 240 consenting adult of ASA class I and II and in the age range from 20 to 65 years of either sex, scheduled for routine surgery under general anaesthesia were enrolled in this prospective observational study during the period of June 2015 to May 2016. Patients with upper airway deformity, recent head and neck surgery, ASA class III and IV, and BMI $\geq$ 30 were excluded. Preoperatively each patient's airway was evaluated on AASI, ULBT and RHTMD.

Taking the observed sensitivity of UBLT, RHTMD and AASI for identifying DVL as $52.38 \%, 38.10 \%$, and $66.67 \%$ respectively, and their specificity was $85.98 \%, 77.34 \%$, and $98.44 \%$ respectively with prevalence DVL as $18.43 \%{ }^{7}$ The estimated sample size with the desired precision of $15 \%$ and $5 \%$ level of significance was 234 patients. To reduce the margin of error, the total sample size of 240 was taken.

Calculation of AASI ${ }^{4}$-(Figure 1)

With the patient in a supine position and arms by the sides of the body, the AASI was calculated

(1) Using a scale, vertical Line A was drawn from the top of the acromion process to the upper border of the axilla at the pectoralis major muscle.

(2) Horizontal Line B was drawn from suprasternal notch to meet line A and

(3) The Line $\mathrm{C}$ will be part of line A that lies above the point at which line $\mathrm{B}$ meet line $\mathrm{A}$

So, AASI is a ratio of the length of line $\mathrm{C}$ to Line $\mathrm{A}(\mathrm{AASI}=\mathrm{C} / \mathrm{A})$

Measurement of RHTMD- The patient in a supine position and arms by the sides of the body with head extended, thyromental distance (TMD) was measured from the centre of the mentum to thyroid notch. Then the ratio of the height of patient to TMD will be RHTMD.

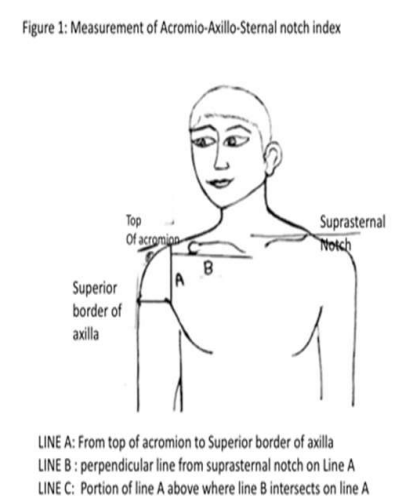

Measurement of ULBT- With patient either in supine or sitting position and arms by the sides of the body: ULBT is classified Class 1-the lower incisors can touch the upper lip above the vermilion line. Class II-lower incisors could touch the upper lip below the vermilion line. Class III-lower incisors could not bite the upper lip.

General anaesthesia was induced as per standard departmental protocol. Direct laryngoscopy was performed by an anaesthesiologist with a minimum of 3 years clinical experience who was also blinded to preoperative airway indices, with head in sniffing position, using appropriately sized Macintosh blade. The best laryngoscopy view was noted according to the Cormack Lehane (CL)grading system. Grade I = full glottis view; Grade II = glottis partly visible, anterior commissure not visualized; Grade III = only epiglottis is visible; Grade IV = no epiglottis visualized.

Visualization of C-L Grades I and II was inferred as an easy visualization of the larynx (EVL) and $\mathrm{C}-\mathrm{L}$ Grades III and IV as DVL. The DVL is synonymous with difficult intubation during laryngoscopy. ${ }^{3}$

If difficult intubation was encountered in the first attempt, intubation was then attempted with adjustment of external laryngeal pressure, the number of attempts, or any other method adopted to intubate was recorded. If airway could not be secured in 3 attempts, it was considered as failed intubation.

Patient data were recorded as mean \pm SD. Quantitative variables were compared using the 
T-test/Mann Whitney test between DVL and EVL. The diagnostic test was done to calculate the sensitivity and specificity of UBLT, RHTMD and AASI. McNemar test was used to compare the sensitivity and specificity of AASI with UBLT and RHTMD. The area under the receiver operating curve (AUC of ROC) was used to find cut off point and to compare AUC of UBLT, RHTMD, and AASI for predicting difficult visualization of laryngoscopy. A $p$ value of $<0.05$ was considered statistically significant. The data was analysed using Statistical Package for Social Sciences (SPSS) 21.

\section{Results}

A total of 240 patients of both sexes (53.3\% were males) requiring endotracheal intubation for general anaesthesia were analysed. DVL was observed in $33(13.7 \%)$ patients in our study. The demographic characteristics based on CormackLehane's view on direct laryngoscopy of patients showed no statistical difference in EVL and DVL. DVL was best assessed by AASI and ULBT. Such association was not observed with $\operatorname{RHTMD}(\mathrm{p}$ value $=0.57)$

(Table1). There was no failed intubation, however, in 8 patients $(3.33 \%)$ intubation was done with videolaryngoscope.

Table 1: Patient characteristics and relation with Cormack-Lehane grade

\begin{tabular}{|l|l|l|l|}
\hline & AASI & ULBT & RHTMD \\
\hline Sensitivity & 93.75 & 50.14 & 75.76 \\
$95 \% \mathrm{Cl}$ & {$[77.97-99.26]$} & {$[29.48-70.79]$} & {$[17.74-68.9]$} \\
\hline Specificity & 98.7 & 87.7 & 47.34 \\
$95 \% \mathrm{Cl}$ & {$[94.7-99.21]$} & {$[82.14-91.4]$} & {$[50.38-84.38]$} \\
\hline $\mathrm{PLR}$ & 3889 & 338 & 144 \\
$95 \% \mathrm{Cl}$ & {$[16.29-92.85]$} & {$[1.98-5.77]$} & {$[1.14-1.81]$} \\
\hline $\mathrm{NLR}$ & 6.0 & 63 & 51 \\
$95 \% \mathrm{Cl}$ & {$[0.02-0.24]$} & {$[0.49-0.89]$} & {$[0.49-0.89]$} \\
\hline $\mathrm{PPV}$ & 86.1 & 35 & 18.66 \\
$95 \% \mathrm{Cl}$ & {$[70.5-95.3]$} & {$[20.63-51.68]$} & {$[12.45-26.3]$} \\
\hline $\mathrm{NPV}$ & 99.02 & 90.5 & 92.45 \\
$95 \% \mathrm{Cl}$ & {$[96.5-99.88]$} & {$[85.67-94.18]$} & {$[85.67-96.69]$} \\
\hline Diagnostic & $97.08 \%$ & $81.25 \%$ & $51.25 \%$ \\
accuracy & & & \\
\hline TP & 31 & 14 & 25 \\
\hline TN & 202 & 181 & 98 \\
\hline FP & 5 & 26 & 109 \\
\hline FN & 2 & 19 & 8 \\
\hline
\end{tabular}

Data is presented as $\mathrm{n}(\%)$ or mean \pm standard deviation EVL- easy visualization of the larynx; DVL- difficult visualization of the larynx; BMI body mass index; CL - Cormack - Lehane grading. AASI - acromio- axillo- suprasternal notch index; RHTDM- ratio of height to thyromental distance; ULBT- upper lip bite test;
ASA- American society of Anaesthesiologists physical status classification

In our study, AASI was observed with the highest predictive values. ULBT with a sensitivity of $\{50.4[29.48-70.79]\}$ and specificity $\{87.7[82.14-91.4]\}$ was better than RHTMD. RHTMD had the least sensitivity and other predictive values. (Table 2)

Table 2: Predictive values for AASI, ULBT and RHTMD to predict DVL on C-L grade

\begin{tabular}{|l|l|l|l|l|}
\hline & & \multicolumn{2}{|l}{ CL } & \multirow{2}{*}{ P value } \\
\cline { 3 - 4 } & Total & Easy & Difficult & \\
\hline Gender & & & & \\
\hline MALE & $128(53.33 \%)$ & $111(53.62 \%)$ & $17(51.52 \%)$ & 0.822 \\
\hline FEMALE & $112(46.67 \%)$ & $96(46.38 \%)$ & $16(48.48 \%)$ & \\
\hline $\begin{array}{l}\text { AGE } \\
\text { (years) }\end{array}$ & $39.02 \pm 14.68$ & $38.61 \pm 14.57$ & $41.58 \pm 15.35$ & 0.275 \\
\hline $\begin{array}{l}\text { WEIGHT } \\
(\mathrm{Kg})\end{array}$ & $59.25 \pm 14.14$ & $58.76 \pm 14.02$ & $62.33 \pm 14.74$ & 0.337 \\
\hline $\begin{array}{l}\text { HEIGHT } \\
(\mathrm{cm})\end{array}$ & $159.05 \pm 8.66$ & $158.99 \pm 9.02$ & $159.46 \pm 6$ & 0.198 \\
\hline $\begin{array}{l}\text { BMI } \\
\left(\mathrm{kg} / \mathrm{m}^{2}\right)\end{array}$ & $23.48 \pm 5.46$ & $23.28 \pm 5.28$ & $24.76 \pm 6.38$ & 0.371 \\
\hline AASI & $0.40 \pm 0.96$ & $0.38 \pm 0.08$ & $0.55 \pm 0.05$ & $<.0001$ \\
\hline RHTMD & $20.96 \pm 3.18$ & $21.09 \pm 3.25$ & $20.12 \pm 2.57$ & 0.157 \\
\hline ULBT & $1.95 \pm 0.62$ & $1.88 \pm 0.6$ & $2.42 \pm 0.5$ & $<.0001$ \\
\hline ASA 1 & $162(67.15 \%)$ & $139(69.7 \%)$ & $23(69.7 \%)$ & \\
\hline ASA II & $78(32.85 \%)$ & $68(32 \%)$ & $10(30.3 \%)$ & 0.77 \\
\hline
\end{tabular}

AASI :acromio-axillo-suprasternal notch index; ULBT: upper lip bite test; RHTMD: ratio of height to thyromental distance; PLR: positive likelihood ratio; NLR: negative likelihood ratio; PPV: positive predictive value; NPV: negative predictive value; TP: true positive; TN: true negative; FP: false positive; FN: false negative; CI: confidence internal

Table 3: Comparison of area under curve ROC of AASI, ULBT, RHTMD

\begin{tabular}{|c|c|c|c|}
\hline & $\begin{array}{l}\text { Area under the ROC } \\
\text { curve }\end{array}$ & P value & Cut-off \\
\hline $\begin{array}{l}\text { AASI } \\
95 \% \mathrm{Cl}\end{array}$ & $\begin{array}{l}0.965 \pm 0.213 \\
0.933-0.98\end{array}$ & $<0.0001$ & $>0.49$ \\
\hline $\begin{array}{l}\text { ULBT } \\
95 \% \mathrm{Cl}\end{array}$ & $\begin{array}{l}0.7202 \pm 0.0365 \\
0.65-0.78\end{array}$ & $<0.0001$ & $>2$ \\
\hline $\begin{array}{l}\text { RHTMD } \\
95 \% \mathrm{Cl}\end{array}$ & $\begin{array}{l}0.576 \pm 0.051 \\
0.511-0.699\end{array}$ & 0.133 & $<21$ \\
\hline
\end{tabular}


Figure 2: Receiver operating curve for AASI in prediction of difficult laryngoscopy

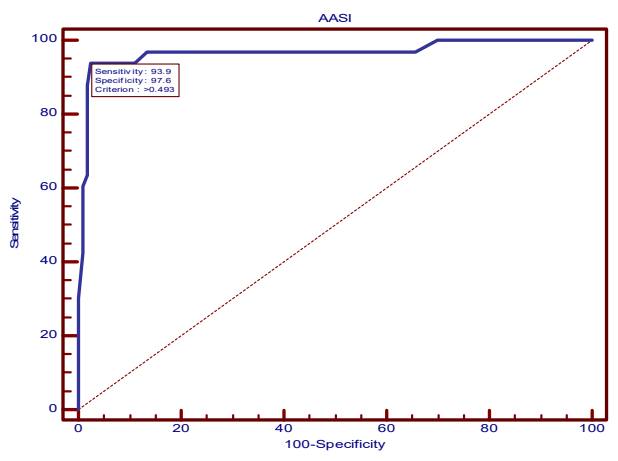

Figure 3: Receiver operating curve for ULBT in prediction of difficult laryngoscopy.

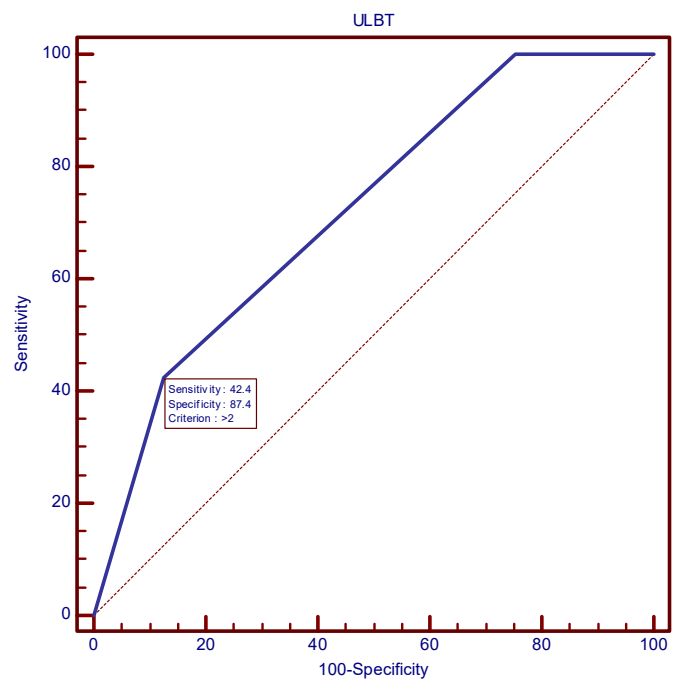

Figure 4: Receiver operating curve for RHTMD in prediction of difficult laryngoscopy

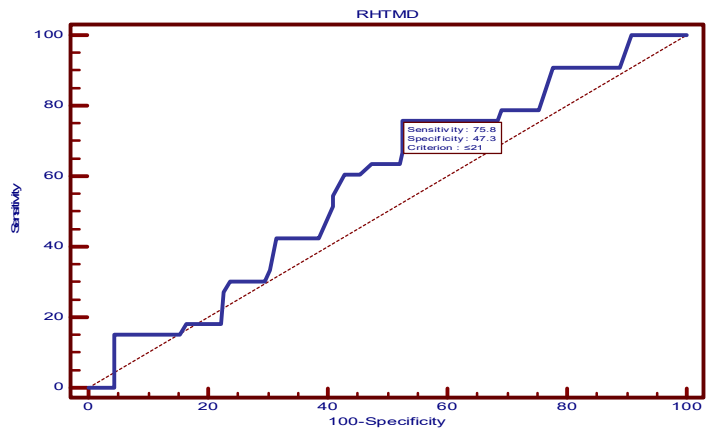

Similarly, the area under ROC for AASI was statistically higher as compared to RHTMD and ULBT.The best cutoff points for predicting difficulty in endotracheal intubation observed as for AASI $>0.49$, RHTMD $<21 \mathrm{~cm}$, ULBT $>$ grade 2 (Table 3, Figure 2,3,4).

\section{Discussion}

To minimize the risk of complications associated with a difficult airway, the screening test should be easy to perform and should have high sensitivity, specificity and high positive predictive values, and low false-positive value. Despite various tests available, none can correctly predict difficult intubation with $100 \%$ accuracy.

Although difficulty in laryngoscopy was found in $13.7 \%$, there was no failed intubation in our study. However, in $3.33 \%$ patients video laryngoscope was used for intubation. Twenty-three patients required external laryngeal pressure and ten patients needed external laryngeal pressure plus stylus for successful intubation.

The incidence of difficult laryngoscopy reported in various studies range from $1.3 \%$, to $13 \%$. $8,9,10$ This variation could be due to the lack of uniformity in grading laryngoscopy views, interobserver error and available facilities.

Thirty-three patients, who had CL grade IIIand IV (DVL), AASI with value $>0.49$ correctly predicted difficulty in 31 patients (true positive), RHTMD $<21 \mathrm{c}$ min 25 patients where as ULBT grade III could predict difficulty in intubation in 14 patients only. Thus, suggesting a strong correlation of higher AASI value with difficult intubation. AASI had PPV of $86.11 \%$ and PLR of 3869 further endorse this that AASI $>0.49$ has a statistically higher ability to predict difficult intubation. Again, the accuracy of AASI was found to be higher than the other tests, which again testifies that the AASI carries lower false positives in predicting a difficult laryngoscopic view.

High sensitivity and specificity of AASI were consistent with the result of previous study. ${ }^{4}$ Sensitivity and specificity in our study was higher than that in the original study and it could be the result of variation in the measurement.

ULBT has been inferred as a simple and useful predictor of difficult intubation. ${ }^{11} \mathrm{~A}$ high diagnostic accuracy (81.5\%), and low falsepositive testifies that ULBT could reasonably be a good bedside test for prediction of difficult intubation. Our result for ULBT was comparable to some previous studies. ${ }^{11,12}$ However, 
ULBT has some limitation as to the inability of the patient to understand how to perform correctly and variation in cranio facial configuration among the population. ${ }^{13}$

In our study, sensitivity (75.6\%) and specificity (47.34\%) of RHTMD was in concurrence with other studies. ${ }^{14,15}$ The low specificity and PPV of RHTMD, in our study, can be explained by a large number of false-positive cases (109 out of 240). Assessment done by RHTMD depends on the patients' thyromental distance and the height, so the cut-off point may vary with the ethnicity and inter-observer error while measuring.

In our study, patients who had AASI $<0.49 \mathrm{cms}$ proved that the chances of difficult intubation were very low. Thus, higher NPV of AASI in our study suggests that the test correlated well with the ease of laryngoscopy and adequately eliminated patients with difficult intubation. ULBT with a specificity of $87 \%$ showed that ULBT I and II can also be a good predictor of easy intubation. On comparing, unlike AASI and ULBT, RHTMD had the least correlation with the laryngeal view.

The results of our study validate the superiority of AUC for AASI over ULBT and RHTMD for its efficacy in predicting difficult laryngoscopy. AUC of ROC for AASI (AUC $=0.89$ ) was large as documented in the original study. ${ }^{7}$ We observed the best predictive cut-off point for predicting difficulty with endotracheal intubation for AASI as $>0.49 \mathrm{cms}$, RHTMD $<.21 \mathrm{~cm}$, ULBT $>2$. AASI value of less than $0.49 \mathrm{cms}$ is regarded as EVL and value more than $0.49 \mathrm{cms}$ is taken as criteria for DVL.

The limitation of our study was that it included non-obese adult north Indian population, so may not be reliable for other populations because of ethnic variation. Also, further studies with bigger sample size and also studies using AASI in comparing alone and in combination with other tests of airway assessment viz, sternomental distance, neck circumference, hyomental distance is needed to establish the usefulness of AASI in predicting the difficult airway.

\section{Conclusion}

AASI with high sensitivity, specificity, predictive value, and low false negativity, is a sensitive, easy bedside test to identify a difficult airway in the normal adult population for surgery under general anaesthesia especially when the cut off $>0.49$.

\section{References}

1. Tiret L, Desmonts JM, Hatton F, Vourc'h G. Complications associated with anaesthesia - a prospective survey in France. Can Anaesth Soc J 1986;33(3):336-44.

https://doi.org/10.1007/BF03010747 PMid:3719435

2. Benumof JL, Scheller MS. The importance of transtracheal jet ventilation in the management of the difficult airway. Anesthesiology 1989;71:769. https://doi.org/10.1097/00000542-19891100000023

PMid:2683873

3. Benumof JL. Difficult laryngoscopy: Obtaining the best view. Can J Anaesth 1994; 41:361

https://doi.org/10.1007/BF03009856 PMid:8055601

4. Kamranmanesh MR, Jafari AR, Gharaei B, Aghamohammadi H, Poor ZamanyN.k. M, Kashi AH. Comparison of acromioaxillosuprasternal notch index (a new test) with modified Mallampati test in predicting difficult visualization of larynx. Acta AnaesthesiolTaiwanica. 2013;51(4):141-4. https://doi.org/10.1016/j.aat.2013.12.001 PMid:24529668

5. Khan ZH, Kashfi A, Ebrahimkhani E. A comparison of the upper lip bite test (a simple new technique) with modified Mallampati classification in predicting difficulty in endotracheal intubation: A prospective blinded study. AnesthAnalg. 2003;96(2):595-9.

https://doi.org/10.1213/00000539-200302000$\underline{00053}$

6. Safavi M, Honarmand A, Hirmanpour A, sheikhani Q, JalaliH.Acromio-AxilloSuprasternal Notch Index: A new screening test to predict difficult laryngoscopy in general population. J.Anesth Surg.2016;3(4):1-6 https://doi.org/10.15436/2377-1364.16.041

7. Ramadhani SAL, Mohamed LA, Rocke DA, Gouws E. Sternomental distance as the sole predictor of difficult laryngoscopy in obstetric anaesthesia. $\mathrm{Br} \mathrm{J}$ Anaesth. 1996;77:312-6. https://doi.org/10.1093/bja/77.3.312

PMid:8949801 
8. Wilson M, Spiegelhalter D, Robertson J, Lesser P. Predicting difficult intubation 2. Br J Anaesth. 1988;61:211-6.

https://doi.org/10.1093/bja/61.2.211

PMid:3415893

9. Yamamoto K, Tsubokawa T, Shibata K, Ohmura S, Nitta S, Kobayashi T. Predicting difficult intubation with indirect laryngoscopy. Anesthesiology. 1997;86:316-21.

https://doi.org/10.1097/00000542-199702000-

$\underline{00007}$

PMid:9054250

10. Khan ZH, Kashfi A, Ebrahimkhani E. A comparison of the upper lip bite test (a simple new technique) with modified Mallampati classification in predicting difficulty in endotracheal intubation: A prospective blinded study. AnesthAnalg. 2003;96(2):595-9. https://doi.org/10.1213/00000539-200302000$\underline{00053}$

11. Eberhart LHJ, Arndt C, Cierpka T, Schwanekamp J, Wulf H, Putzke C. The reliability and validity of the Upper Lip Bite test compared with the Mallampati classification to predict difficult laryngoscopy: An external prospective evaluation. AnesthAnalg. 2005;101:284-9. https://doi.org/10.1213/01.ANE.0000154535.334 $\underline{29.36}$

PMid:15976247

12. Hester CE, Dietrich SA, White SW, Secrest JA, Lindgren KR, Smith T. A comparison of preoperative airway assessment techniques: The modified Mallampati and the upper lip bite test. AANA J. 2007;75:177-82.

13. Safavi M, Honarmand A, Zare N. A comparison of the ratio of patient's height to thyromental distance with the modified Mallampati and the upper lip bite test in predicting difficult laryngoscopy. Saudi J Anaesth2011;5:258-63. t https://doi.org/10.4103/1658-354X.84098 PMid:21957403 PMCid:PMC3168341

14. Safavi M, Honarmand A, Amoushahi M. Prediction of difficult laryngoscopy: Extended mallampati score versus the MMT, ULBT and RHTMD. Adv Biomed Res. 2014;3:133.

https://doi.org/10.4103/2277-9175.133270

PMid:24949304 PMCid:PMC4063103 